\title{
Recent dibaryon studies at ANKE
}

D. Tsirkov ${ }^{1, *}$, V. Komarov ${ }^{1}$, T. Azaryan ${ }^{1}$, S. Dymov ${ }^{2}$, A. Kunsafina ${ }^{1,3,4}$, V. Kurbatov ${ }^{1}$, Zh. Kurmanaliyev ${ }^{1,3,4}$, and Yu. Uzikov ${ }^{1,5,6}$

for the ANKE Collaboration

${ }^{1}$ Laboratory of Nuclear Problems, Joint Institute for Nuclear Research, RU-141980 Dubna, Russia

${ }^{2}$ Institut für Kernphysik, Forschungszentrum Jülich, D-52425 Jülich, Germany

${ }^{3}$ Al-Farabi Kazakh National University, KZ-050040 Almaty, Kazakhstan

${ }^{4}$ Institute of Nuclear Physics, KZ-050032 Almaty, Kazakhstan

${ }^{5}$ Dubna State University, RU-141980 Dubna, Russia

${ }^{6}$ Department of Physics, Moscow State University, RU-119991 Moscow, Russia

\begin{abstract}
The recent results of dibaryon studies at ANKE are being presented, along with our plans concerning further studies on the topic. The $D_{03}$ resonance has been observed at ANKE in the coherent $p d \rightarrow p d+(\pi \pi)^{0}$ channel, accompanied by the $\mathrm{ABC}$ effect. An explanation of the $\mathrm{ABC}$ effect has been proposed, suggesting to to explain it as a kinematic cumulation of the pion pairs in the $D_{03} \rightarrow D_{12}+\pi \rightarrow d+(\pi+\pi)_{I=0}$ cascade decay channel due to the predominantly collinear $D_{12}$ decay. Based on this hypothesis, a search for a $D_{21}$ signal in the $p p \rightarrow\{p p\}_{s} \pi \pi$ reaction is suggested. The $p$-wave dibaryon resonances ${ }^{3} P_{2}$ and ${ }^{3} P_{0}$ have been observed at ANKE; for ${ }^{3} P_{0}$ it is a first experimental observation. Preliminary results for a resonance peak in the $p p \rightarrow\{p p\}_{s} \pi^{0}$ reaction with the mass $m=2646 \pm 5 \mathrm{MeV}$ and the width $\Gamma=132 \pm 14 \mathrm{MeV}$ are being presented. Also we propose to search for the narrow $N N^{*}(1535)$ resonance in the $p d \rightarrow p d \eta$ reaction.
\end{abstract}

\section{Introduction}

Derivation of the hadron spectroscopy from QCD laws has been a important challenge for decades, but theoretical predictions cannot yet provide a clear set of rules for hadron construction. One of the ways to advance in this field is to study exotic hadrons such as tetra-quarks, penta-quarks, and hexa-quarks. They are allowed by QCD, but convincing experimental observations of tetra-quarks and penta-quarks have been made only recently (see, e. g., $[1,2]$ and refs. therein). As for hexa-quarks, a search for them has already a long history.

\section{$2 \mathrm{SU}(6)$ dibaryon classification}

Resonances in two-baryon systems were first considered in [3] and developed in [4] soon after the SU(3) symmetry formulation. In Ref. [4] a classification has been introduced for non-strange two-baryon states as members of an SU(6) unitary multiplet. The members of the multiplet followed the notation $D_{I J}$, where $I$ is the isospin and $J$ is the angular momentum.

\footnotetext{
*e-mail: cyrkov@jinr.ru
} 
The lowest states of the multiplet were the deuteron $D_{01}$, and the deuteron virtual singlet state or the $s$-wave proton pair $D_{10}$. The next, higher states, so far unobserved that time, were the $s$-wave $N \Delta(1232)$ resonance $D_{12}$, the $s$-wave $\Delta(1232) \Delta(1232)$ resonance $D_{03}$, the charge-3 $p \Delta^{++}(1232)$ resonance $D_{21}$ and the charge- $4 \Delta^{++}(1232) \Delta^{++}(1232)$ resonance $D_{30}$.

The extensive search for two-baryon states began later after the development of the quarkbag models, that reproduced quite well the spectroscopy of the known hadrons and predicted two-baryon resonances $[5,6]$. In these searches, the term "dibaryons" was used to denote "genuine" compact six-quark resonances, and a main criterion of their existence was a small width, $\Gamma \lesssim 100 \mathrm{MeV}[7,8]$.

Numerous studies have led to the discovery of three resonance states ${ }^{1} D_{2},{ }^{3} F_{3},{ }^{3} P_{2}$ in the partial wave analysis (PWA) of the $p p \rightarrow p p$ elastic scattering and then of the $p p \rightarrow$ $d \pi^{+}$reaction (see [9-11] and refs. therein). Here the notation follows the PWA standard ${ }^{2 S+1} L_{J}$, where $L$ is the orbital angular momentum, $S$ is the spin, and $J$ is the total angular momentum. The quantum numbers of the ${ }^{1} D_{2}$ two-baryon resonance state coincide with those of the Dyson's $D_{12}$ dibaryon, and the predicted $D_{12}$ mass, $\sim 2176 \mathrm{MeV}$, is close to the ${ }^{1} D_{2}$ mass, 2120-2150 MeV according to different PWA solutions [12].

However, the dominant interpretation for these resonances was that they are conventional hadron states in the $\Delta N$ channel instead of "genuine" quark dibaryons, since their $S$-matrix poles were found to be close to the $\Delta N$ branching line, and their widths close to that of $\Delta(1232)$. The situation has changed after the observation of an isoscalar two-baryon resonance state in the quasi-free channel $p n \rightarrow p n \pi^{0} \pi^{0}$, with a mass $2370-2380 \mathrm{MeV}$ and an exceptionally small width $\Gamma \approx 70 \mathrm{MeV}$ [13-15], that was suggested as a candidate for the "true" dibaryon. The mass and the quantum numbers of this state [14] allowed to associate it with the Dyson's $D_{03}$ dibaryon.

Calculations within constituent-quark models predicting the $D_{03}$ dibaryon have a long history, and recent development of the model $[16,17]$ has resulted in the successful calculation of its mass, total width and partial decay widths. It follows from these works that the resonance wave function has a two-component content: a $\Delta \Delta$ part and a dominant hidden-color component, the six-quark exotic state. Nonetheless, the traditional meson-baryon approach to describe this resonance is still up to date. In [18] a general motivation was suggested for the decrease of the $D_{03}$ width compared to that of the free $\Delta(1232)$. The recent work $[19,20]$ considered the two-component structure of the $D_{03}$ resonance, consisting of a compact $\Delta \Delta$ component and a loose $N \Delta$ near-threshold system, and succeeded in describing its mass, total and partial decay widths. Thus, the resonance behavior of the $p n \rightarrow p n \pi^{0} \pi^{0}$ cross section can be rather well reproduced by both the meson-baryon and the quark-model approaches. On the basis of that, we would use the term "dibaryon" for any hadronic system with the baryon number two and a resonance-like behavior, without implying its "genuine" quark structure.

Recently, a signal for the $D_{21}$ dibaryon has been observed as well at WASA@COSY [21]. The quasi-free $p p \rightarrow p p \pi^{+} \pi^{-}$reaction has been measured exclusively in $p d$ collisions, allowing to obtain total and differential cross sections for the energy region $\sqrt{s}=2350-2460 \mathrm{MeV}$, which covers $D_{21}$ mass expected from the original predictions [4]. The measured cross sections are significantly underpredicted by the $t$-channel "modified Valencia" calculations ignoring the $D_{21}$ dibaryon, while introducing the one with the mass $m=2140 \mathrm{MeV}$ and the width $\Gamma=110 \mathrm{MeV}$ to the model allows to get rid of this discrepancy.

\subsection{ANKE observations}

In all the experiments performed so far the $D_{03}$ dibaryon was excited via free or quasi-free collision of fast nucleons. We at ANKE observed $D_{03}$ dibaryon in the coherent $p d \rightarrow p d+$ $(\pi \pi)^{0}$ channel where both nucleons of the deuteron were excited to $D_{03}$ by the $t$-channel 
meson exchange with a fast projectile proton [22]. The reaction $p+d \rightarrow p+d+X$ was studied at $0.8-2.0 \mathrm{GeV}$ proton beam energies, and the proton-deuteron pairs emerging with high momenta, $0.6-1.8 \mathrm{GeV} / c$, were detected at small angles with respect to the proton beam. The invariant mass of the $d \pi \pi$ system in the region of pion-pair production exhibits a resonancelike peak at $m_{d \pi \pi} \approx 2360 \mathrm{MeV} / c^{2}$ with the width $\Gamma \approx 100 \mathrm{MeV} / c^{2}$, corresponding to the $D_{03}$ excitation.

The $D_{03}$ is known to have an association to the ABC effect-a significant near-threshold enhancement in the spectrum of the pion pair invariant mass $m_{\pi \pi}$, observed in the reactions with production of the isoscalar pion pair accompanied by the bound light nucleus, at the position $m_{\pi \pi} \approx 300 \mathrm{MeV} / c^{2}$ with a surprisingly small width about $40 \mathrm{MeV} / c^{2}$ [23]. At ANKE, the invariant mass of the pion pair $m_{\pi \pi}$ also reveals a local enhancement with a structure of a narrow bump placed above a smooth continuum, specific to the $\mathrm{ABC}$ effect.

The explanation of the ABC effect, especially a rather narrow width of the enhancement, has puzzled science for decades. Various explanations have been proposed (see, e. g., [23, 24]). The one that seemed promising for us was the cascade decay channel

$$
D_{03} \rightarrow D_{12}+\pi \rightarrow d+(\pi+\pi)_{I=0},
$$

proposed in [24]. That work suggested that the effect could arise from the interference between (1) and another $D_{03}$ decay channel,

$$
D_{03} \rightarrow d+\sigma \rightarrow d+(\pi+\pi)_{I=0},
$$

with the mass and the width of the $\sigma$ meson equal to $m_{\sigma}=300 \mathrm{MeV}$ and $\Gamma_{\sigma}=100 \mathrm{MeV}$ due to the hypothetical chiral symmetry restoration in the hot and dense quark matter composing the $D_{03}$ dibaryon. Since such restoration in the case of the $D_{03}$ resonance decay does not seem to have enough confirmations to us, we have proposed a hypothesis that the $\mathrm{ABC}$ effect is a kinematic effect of pion-pair cumulation near the specific excitation energy in channel (1) due to the binary character of the reactions composing the cascade and predominantly collinear decay of the intermediate $D_{12}$ dibaryon [22]. At the extreme case of zero dibaryon widths and exactly collinear decay, the position of the ABC enhancement would be the $\delta$ function at $m_{\pi \pi}=271 \mathrm{MeV} / c^{2}$ for $\pi^{0} \pi^{0}$. Both receding from the collinear kinematics and dispersion of the resonance masses cause the peak smearing, but a simple simulation with the standard dibaryon masses and a $\cos \theta$ angular distribution for the $D_{12}$ decay gives a peak position similar to that of the ABC one [22]. One more argument in favor of the collinear character of the $D_{12}$ decay is the concentration of fast deuterons, ${ }^{3} \mathrm{He},{ }^{4} \mathrm{He}$ nuclei near $0^{\circ}$ and $180^{\circ}$ angles in the experiments where the narrow $\mathrm{ABC}$ enhancement was observed [13, 22, 25].

One of the common reactions where it is suggested to search for the $D_{21}$ dibaryon is the $p p \rightarrow D_{21} \pi^{-} \rightarrow p p \pi^{+} \pi^{-}$channel $[4,21]$. The effect of the pion-pair cumulation could take place in this reaction as well if we select final proton pairs with a small excitation energy, which would lead to a quasi-binary kinematics in the second step of the cascade. A simple calculation, done for the case of zero dibaryon width and exactly collinear decay, shows that the position of the two-pion peak would depend on the initial energy (see Fig. 1), though in the beam energy region $T_{p}=1-2 \mathrm{GeV}$ it would be close to the threshold, resulting in the ABC-like peak.

Fortunately, these are ANKE data on the $p p \rightarrow\{p p\}_{s}+(\pi \pi)^{0}$ reaction at $T_{p}=1.1$ and $1.4 \mathrm{GeV}$ [26], that show a prominent near-threshold enhancement. The model describing the data proposed in that paper describes the data well in the whole $\pi \pi$ mass range except the region near the threshold, where the data seem to show some excess over the model predictions. We suggest to estimate the near-threshold enhancement numerically and to consider, whether it could be a sign for the ABC-like effect associated with the $D_{21}$ production. 


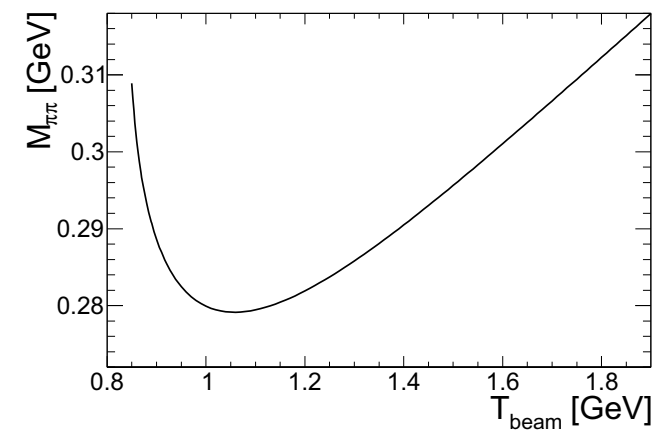

Figure 1. Position of the $\pi^{+} \pi^{-}$enhancement calculated for the reaction $p p \rightarrow D_{21} \pi^{-} \rightarrow p p \pi^{+} \pi^{-}$in the approximation of zero dibaryon width and exactly collinear decay.

\section{Dibaryons outside the SU(6) scheme}

Known dibaryons are not limited to the Dyson's classification. The resonance states ${ }^{3} P_{2}$ and ${ }^{3} F_{3}$ have long been known from PWA of the $p p$ scattering [9-11]. According to the $D_{I J}$ notation, ${ }^{3} P_{2}$ would correspond to $D_{12}$ with negative parity, $D_{12}^{-}$(all SU(6) dibaryons have positive one), and ${ }^{3} F_{3}$ would be $D_{13}^{-}$.

Since ${ }^{3} P_{2}$ is the least intensive resonance of the ones obtained from PWA, uncertainties in its parameters are rather large. New opportunities to study this resonance are offered by the

$$
p p \rightarrow\{p p\}_{s}+\pi^{0}
$$

channel (here diproton $\{p p\}_{S}$ is a proton pair in ${ }^{1} S_{0}$ state), since the intensive ones ${ }^{1} D_{2},{ }^{3} F_{3}$ are forbidden in it. At ANKE we have measured for the first time the differential cross section $d \sigma / d \Omega$ and the analyzing power $A_{y}$ in the $\Delta(1232)$ excitation energy region of the reaction (3) (see [27] and refs. therein). The data exhibited the following features: a prominent peak in the energy dependence of the forward differential cross section in the region of $\Delta(1232)$ excitation, the anomalous dip for the differential cross section at zero angle, and the significant analyzing power. A simple PWA of the ANKE results together with the data from [28] has been performed, taking into account only the transitions ${ }^{3} P_{0} \rightarrow{ }^{1} S_{0} s$ and ${ }^{3} P_{2} \rightarrow{ }^{1} S_{0} d$, since the other ones are known to be small from the PWA of the $p p \rightarrow p p$ and $p p \rightarrow d \pi^{+}$reactions. This allowed to explain the aforementioned features observed for the data as a result of the interference for the ${ }^{3} P_{2}$ and ${ }^{3} P_{0}$ resonances.

The PWA results also allowed specify the parameters of the ${ }^{3} P_{2}$ resonance, that turned out to be $m=2195 \pm 8 \mathrm{MeV} / c^{2}$ and $\Gamma=134 \pm 22 \mathrm{MeV} / c^{2}$. Besides, a ${ }^{3} P_{0}$ was observed experimentally for the first time, and PWA gave its mass $m=2199 \pm 5 \mathrm{MeV} / c^{2}$ and width $\Gamma=94 \pm 11 \mathrm{MeV} / c^{2}$. In the $D_{I J}$ notation the ${ }^{3} P_{0}$ resonance would be $D_{10}^{-}$, that could be treated as an excited state of the $s$-wave diproton / virtual singlet deuteron $D_{10}^{+}$. Both ${ }^{3} P_{2}$ and ${ }^{3} P_{0}$ resonances belong to the new family of $p$-wave dibaryons, as opposed to the $s$-wave dibaryons considered earlier.

One of the first ANKE studies of the $p p \rightarrow\{p p\}_{s} \pi^{0}$ reaction [29] suggested the following feature of the energy spectrum of the forward cross section: after reaching a minimum at $T_{p}=1.4 \mathrm{GeV}$, it increased again for $T_{p}=2.0 \mathrm{GeV}$, while its angular slope changed the sign. That suggested the existence of the second peak in the cross section spectrum at the energies $T_{p}>1.4 \mathrm{GeV}$. We have preliminarily analyzed the data on this reaction at several energies in the $T_{p}=1-3 \mathrm{GeV}$ range, which revealed the clear second peak with the mass $m=2646 \pm 5 \mathrm{MeV}$ and the width $\Gamma=132 \pm 14 \mathrm{MeV}$. Whether this peak could be interpreted as a sign for some dibaryon resonance, for example $N \Delta(1700)$ or $\Delta(1232) N^{*}(1440)$, is subject to further studies. 


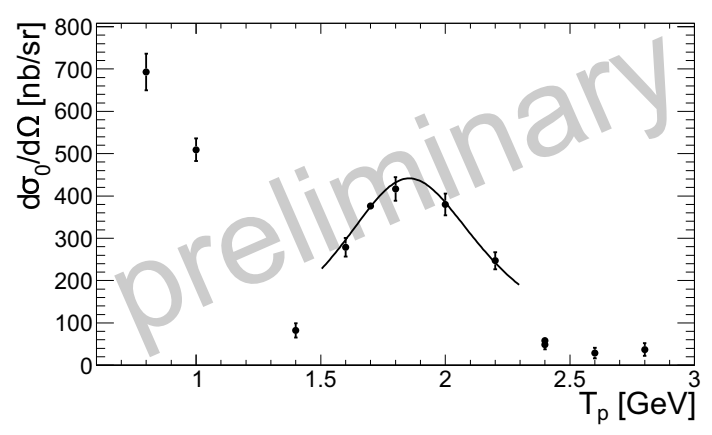

Figure 2. Energy dependence of the differential cross section $d \sigma / d \Omega$ at zero angle for the reaction $p p \rightarrow\{p p\}_{s} \pi^{0}$. The curve is a Breit-Wigner fit to the data with the mass $m=2646 \pm 5 \mathrm{MeV}$ and the width $\Gamma=132 \pm 14 \mathrm{MeV}$.

The analysis of data on the $p d \rightarrow p d+X$ reaction in the region of the $\eta$ meson production exhibits a strong narrow enhancement in the event distribution with respect to mass of the $d \eta$ system (Fig. 3). Preliminary fitting of the Breit-Wigner function plus constant background to this data gives the position of the peak $m_{0}=2432 \pm 2 \mathrm{MeV} / c^{2}$ an its width $\Gamma=20 \pm 3 \mathrm{MeV} / c^{2}$. The mass suggests that it could be a signal for the resonance state of the nucleon and the $N^{*}$ (1535) baryon, that is known to decay predominantly to $N \eta$ [30]. In this case, it would be a first dibaryon including a non- $\Delta$ excited baryon with a dominant decay channel involving a meson other than $\pi$. Due to the fact that the peak is located very close to the acceptance boundary, a evaluation of the setup acceptance is required for this data to provide convincing results. It is planned to be done in the nearest future. Besides, we are going to examine data on the $p d \rightarrow p d+X$ reaction from other ANKE beam times for the search of this signal in the more favorable conditions.

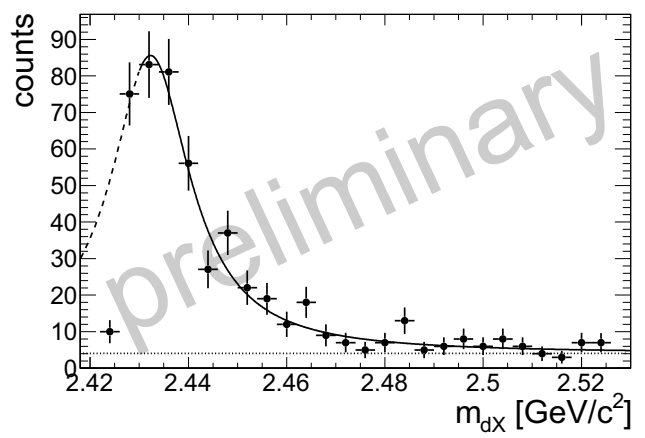

Figure 3. Event counts with respect to the invariant mass of the $d \eta$ system $m_{d X}$ for the $p d \rightarrow p d+X$ reaction in the missing mass region $295 \mathrm{MeV} \leq m_{X}^{2}<310 \mathrm{MeV}$. The curve is a fit by the Breit-Wigner function plus constant background $\operatorname{bg}+N\left(\Gamma^{2} / 4\right) /\left[\left(m_{d X}-m_{0}\right)^{2}+\Gamma^{2} / 4\right]$. Its parameters are $N=82 \pm 8$, $m_{0}=2432 \pm 2 \mathrm{MeV} / c^{2}, \Gamma=20 \pm 3 \mathrm{MeV} / c^{2}$, and $\mathrm{bg}=4 \pm 1$.

\section{Conclusion}

- The observation of the $D_{03}$ resonance has been performed at ANKE in the coherent $p d \rightarrow$ $p d+(\pi \pi)^{0}$ channel [22], accompanied by the ABC effect.

- We proposed an explanation of the $\mathrm{ABC}$ effect as a result of kinematic cumulation of the pion pairs in the $D_{03} \rightarrow D_{12}+\pi \rightarrow d+(\pi+\pi)_{I=0}$ decay channel due to the predominantly collinear $D_{12}$ decay.

- We suggest to extract a signal for $D_{21}$ from the $p p \rightarrow\{p p\}_{s} \pi \pi$ data [26] supposing the ABC "cumulation" hypothesis.

- The parameters of the ${ }^{3} P_{2}$ dibaryon resonance have been specified at ANKE, and the ${ }^{3} P_{0}$ dibaryon resonance has been observed for the first time in the $p p \rightarrow\{p p\}_{s} \pi^{0}$ reaction [27]. 
- Preliminary results have been obtained for the resonance peak in the $p p \rightarrow\{p p\}_{s} \pi^{0}$ reaction with the mass $m=2646 \pm 5 \mathrm{MeV}$ and the width $\Gamma=132 \pm 14 \mathrm{MeV}$.

- Preliminary data suggest an existence of the $N N^{*}(1535)$ resonance in the $p d \rightarrow p d \eta$ reaction. Further analysis of the data on this reaction is planned.

We are grateful to colleagues from the COSY team for providing favorable data-taking conditions. Valuable discussions with L. Alvarez-Ruso, V. Baru, A. Gal, Ch. Hanhart, A. Kudryavtsev, V. Kukulin, and J. Niskanen are acknowledged. We are also thankful to H. Clement and T. Skorodko for fruitful discussions at the MESON2018 conference. The work was supported in part by the grants of BMBFJINR, COSY FFE and RFBR (09-02-91332) and by the Heisenberg-Landau program.

\section{References}

[1] M.R. Shepherd, J.J. Dudek, R.E. Mitchell, Nature 534, 487 (2016)

[2] H.X. Chen, W. Chen, X. Liu, S.L. Zhu, Phys. Rep. 639, 1 (2016)

[3] R.J. Oakes, Phys. Rev. 131, 2239 (1963)

[4] F.J. Dyson, N.H. Xuong, Phys. Rev. Lett. 13, 815 (1964)

[5] A.T.M. Aerts, P.J.G. Mulders, J.J. de Swart, Phys. Rev. D 17, 260 (1978)

[6] P.J. Mulders, A.T. Aerts, J.J. de Swart, Phys. Rev. D 21, 2653 (1980)

[7] M.P. Locher, M.E. Sainio, A. Švarc, Adv. Nucl. Phys. 17, 47 (1986)

[8] B.L.G. Bakker, I.M. Narodetskii, Adv. Nucl. Phys. 21, 1 (1994)

[9] R.A. Arndt, L.D. Roper, R.A. Bryan, R.B. Clark, B.J. VerWest, P. Signell, Phys. Rev. D 28, 97 (1983)

[10] R.A. Arndt, I.I. Strakovsky, R.L. Workman, D.V. Bugg, Phys. Rev. C 48, 1926 (1993)

[11] R.A. Arndt, W.J. Briscoe, I.I. Strakovsky, R.L. Workman, Phys. Rev. C 76, 025209 (2007)

[12] SAID interactive code, http://gwdac .phys . gwu.edu

[13] M. Bashkanov et al. (WASA@COSY), Phys. Rev. Lett. 102, 052301 (2009)

[14] P. Adlarson (WASA@COSY), Phys. Rev. Lett. 106, 242302 (2011)

[15] P. Adlarson et al. (WASA@COSY), Phys. Rev. Lett. 112, 202301 (2014)

[16] Y. Dong, P. Shen, F. Huang, Z. Zhang, Phys. Rev. C 91, 064002 (2015)

[17] Y. Dong, F. Huang, P. Shen, Z. Zhang, Phys. Rev. C 94, 014003 (2016)

[18] J.A. Niskanen, Phys. Rev. C 95, 054002 (2017)

[19] A. Gal, Phys. Lett. B 769, 436 (2017)

[20] A. Gal, arXiv:1803.08788 [nucl-th] (2018), 1803.08788

[21] P. Adlarson et al. (WASA@COSY), Phys. Rev. Lett. 121, 052001 (2018)

[22] V. Komarov et al. (ANKE@COSY), arXiv:1805.01493 [nucl-ex] (2018)

[23] M. Bashkanov, H. Clement, T. Skorodko, Nucl. Phys. A 958, 129 (2017)

[24] M.N. Platonova, V.I. Kukulin, Phys. Rev. C 87, 025202 (2013)

[25] J. Banaigs, J. Berger, L. Goldzahl, T. Risser, L. Vu-Hai, M. Cottereau, C. Le Brun, Nucl. Phys. B 67, 1 (1973)

[26] S. Dymov et al. (ANKE@COSY), Phys. Rev. Lett. 102, 192301 (2009)

[27] V. Komarov et al. (ANKE@COSY), Phys. Rev. C 93, 065206 (2016)

[28] R. Bilger et al., Nucl. Phys. A 693, 633 (2001)

[29] V. Kurbatov et al. (ANKE@COSY), Phys. Lett. B 661, 22 (2008)

[30] M. Tanabashi et al. (Particle Data Group), Phys. Rev. D 98, 030001 (2018) 